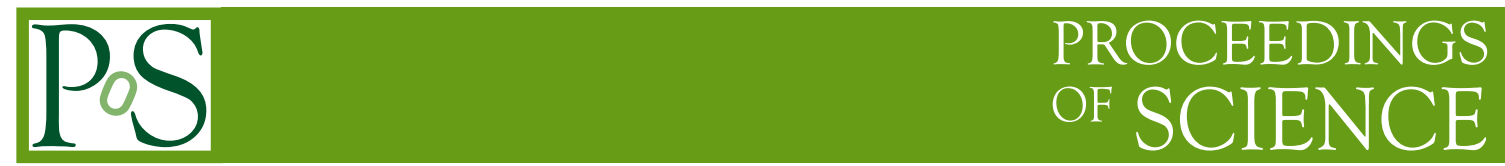

\title{
The Daya Bay Reactor Neutrino Experiment
}

\author{
Yung-Shun Yeh* \\ Institute of Physics, National Chiao Tung University, Hsinchu 30010, Taiwan \\ E-mail: adair.py 94 g@nctu.edu.tw
}

The Daya Bay Reactor Neutrino Experiment, located in Shenzhen, China, is going to probe the last unknown neutrino mixing angle $\theta_{13}$ with a sensitivity of 0.01 or better in $\sin ^{2} 2 \theta_{13}$ at $90 \%$ C.L. through a measurement of the relative rates and energy spectra of reactor anti-neutrinos at different baselines. The Daya Bay Near Hall began taking data on August 16th, 2011, and all of the three experimental halls will be ready for data taking in the summer of 2012. An overview of the experiment and the current status are presented.

The 2011 Europhysics Conference on High Energy Physics-HEP 2011,

July 21-27, 2011

Grenoble, Rhône-Alpes France

${ }^{*}$ On behalf of the Daya Bay Experiment Collaboration 


\section{The Daya Bay Experiment}

The Daya Bay Experiment [1] is located near the Daya Bay nuclear power complex, one of the most powerful nuclear power complexes in the world. Its mission is to probe the last unknown mixing angle $\theta_{13}$ with a sensitivity of 0.01 or better in $\sin ^{2} 2 \theta_{13}$ at $90 \%$ C.L. by measuring the relative rates and energy spectra of reactor anti-neutrinos at different baselines. The survival probability of $\bar{v}_{e}$ as a function of the baseline is given by

$$
P_{e e} \approx 1-\sin ^{2} 2 \theta_{13} \sin ^{2}\left(\frac{\Delta m_{21}^{2} L}{4 E}\right)-\cos ^{4} \theta_{13} \sin ^{2} 2 \theta_{12} \sin ^{2}\left(\frac{\Delta m_{21}^{2} L}{4 E}\right) .
$$

The Daya Bay nuclear power complex has three pair of reactor cores: Daya Bay, Ling Ao I and II. With total thermal power output of $17.4 \mathrm{GW}$, it gives a prolific source of reactor anti-neutrinos. The mountain terrain near the reactor cores provides an ideal site to construct underground laboratories that are well shielded from cosmic rays.

There are three experimental sites in Daya Bay Experiment: Daya Bay Near Site, Ling Ao Near Site, and Far Site. Two near sites, apart from the nuclear power plants by $300 \mathrm{~m}$ and $500 \mathrm{~m}$ respectively, are responsible for measuring the initial fluxes of $\bar{v}_{e}$ from the reactor cores. Far Site is located around the first oscillation maximum region of $P_{e e}$, which is sensitive to $\sin ^{2} 2 \theta_{13}$. Tab. 1 shows the target mass, baselines, overburden, and expected rates for each site.

Table 1: Target mass, baseline, overburden, estimated muon flux and IBD rate at three sites.

\begin{tabular}{|c|c|c|c|}
\hline & Daya Bay site & Ling Ao site & Far site \\
\hline Target mass & 40 tons & 40 tons & 80 tons \\
\hline Baseline & $363 \mathrm{~m}$ & $\begin{array}{c}481 \mathrm{~m} \text { from Ling Ao I } \\
526 \mathrm{~m} \text { from Ling Ao II }\end{array}$ & $\begin{array}{c}1986 \mathrm{~m} \text { from Daya Bay } \\
1615 \mathrm{~m} \text { from Ling Ao I/II }\end{array}$ \\
\hline Overburden & $98 \mathrm{~m}$ & $112 \mathrm{~m}$ & $350 \mathrm{~m}$ \\
\hline Expected Muon flux & $1.2 \mathrm{~Hz} / \mathrm{m}^{2}$ & $0.73 \mathrm{~Hz} / \mathrm{m}^{2}$ & $0.04 \mathrm{~Hz} / \mathrm{m}^{2}$ \\
\hline Expected IBD flux & $840 /$ day $/ \mathrm{module}^{2}$ & $740 /$ day $/ \mathrm{module}$ & $90 / \mathrm{day} / \mathrm{module}$ \\
\hline
\end{tabular}

\section{Anti-neutrino Detector and Muon Veto System}

The anti-neutrino detector(AD) is a three-zone cylindrical structure. Two acrylic vessels with diameters $3 \mathrm{~m}$ and $4 \mathrm{~m}$ are nested inside a 5-m diameter stainless steel vessel(SSV). The inner acrylic vessel(IAV) is filled with Gadolinium doped liquid scintillator as the target region. The medium zone between IAV and the outer acrylic vessel(OAV) is filled with pure liquid scintillator to capture gamma ray escaped from the target region. Mineral oil, filled in the outer-most zone, prevents the external radiation from entering the fiducial volume. There are 1928 -inch photomultiplier tubes mounted around the inner wall of SSV (see Fig. 4). To improve light collection efficiency, reflectors are installed on the top and at the bottom inside the AD. Fig. 1 shows the schematic drawing of the AD.

Each near site has two ADs, while four ADs are deployed in Far site in order to increase the statistics. Each $\mathrm{AD}$ is immerged in the water pool. The water pool shields the backgrounds from 
Figure 1: Schematic drawing of the Daya Bay AD module.

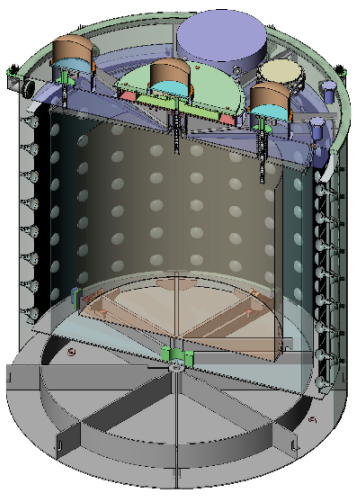

Figure 2: The layout of near sites.

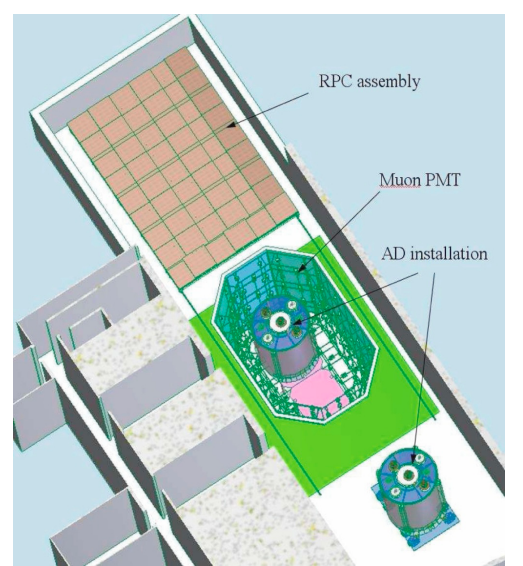

surrounding rocks and serves as a water Cherenkov detector to tag cosmic muons. On the top of the water pool, a layer of resistance plate chambers(RPCs) gives an additional muon-tagging. Combining the water Cherenkov detector and RPC, we expect the muon detection efficiency to be $(99.50 \pm 0.25) \%$. Fig. 2 shows the layout of a near site. Far site has a similar layout with a larger water pool and RPC module array.

\section{Current Status}

Before filling the ADs, full chain test consisting of the first two assembled ADs, automatic calibration units, electronics, trigger and DAQ, has been performed without liquid filled. The results [2] show that both ADs are fully functional.

On August 16th, 2011, the Daya Bay near site began to take data. Fig. 3 shows the status of the Daya Bay near site. All experimental sites will be ready for data taking in the summer of 2012.

Figure 3: The Daya Bay site

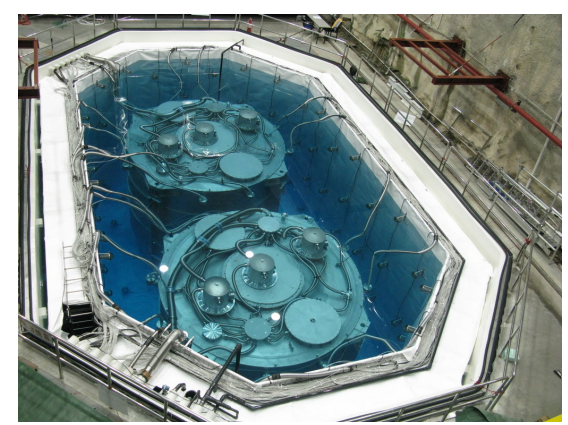

Figure 4: Inside view of the AD

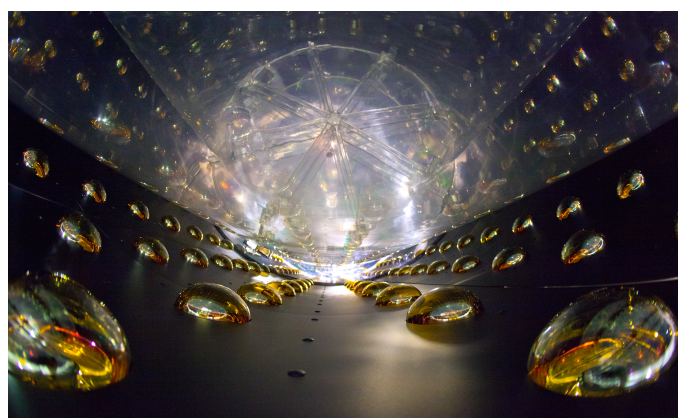

\section{References}

[1] X. Guo et al. [Daya-Bay Collaboration], arXiv:hep-ex/0701029.

[2] Z. Wang [on behalf of the Daya Bay Collaboration], arXiv:1109.3253 [physics.ins-det]. 